Aberdeen Royal Infirmary,

Foresterhill, Aberdeen, Scotland: Infection Unit

A R Mackenzie

R B S Laing

J G Douglas

C C Smith

Department of

Haematology

M Greaves

Correspondence to: Dr A R Mackenzie, Infection Unit, Aberdeen Royal Infirmary, Foresterhill, Aberdeen, Scotland AB25 2ZB, UK

(email: a.r.mackenzie@

arh.grampian.scot.nhs.uk)

Submitted 8 December 1999 Accepted 22 March 2000

\title{
High prevalence of iliofemoral venous thrombosis with severe groin infection among injecting drug users in North East Scotland: successful use of low molecular weight heparin with antibiotics
}

\author{
A R Mackenzie, R B S Laing, J G Douglas, M Greaves, C C Smith
}

\begin{abstract}
Injecting drug use, mainly of heroin, currently represents a major public health issue in the North East of Scotland. The recent tendency of the committed injecting drug user to inject into the groin has created novel problems for the Infection Unit.

Data are presented on 20 consecutive patients admitted between 1994 and 1999 with iliofemoral venous thromboses, often complicated by severe soft tissue infections and bacteraemia as a result of heroin injection into the femoral vein. Nine had coexistent groin abscesses, four had severe streptococcal soft tissue infection of the right thigh, groin and lower abdomen, and two had coincidental soft tissue infections of the upper limb. Nine were bacteraemic on admission. All of the patients were chronic injecting drug users with a median injection duration of 6.5 years. The 18 patients tested for hepatitis $C$ virus were all seropositive. None of the 14 patients tested was positive for HIV.

Seventeen patients were treated with subcutaneous low molecular weight heparin (tinzaparin), three having received intravenous unfractionated heparin initially. The tinzaparin was self administered and given for a median duration of seven weeks. One patient declined to have any treatment. Three months after presentation eight patients were asymptomatic, seven had a persistently swollen leg, and five were lost to follow up. None developed clinically apparent pulmonary embolism after institution of anticoagulant therapy.
\end{abstract}

The management of iliofemoral venous thrombosis in injection drug users is problematic because of poor venous access, non-compliance with prescribed treatment, ongoing injecting behaviour, and coexistent sepsis. It is unlikely that a randomised trial of standard treatment with heparin and warfarin versus low molecular weight heparin alone would be practical in this patient group. These retrospective data indicate that the use of tinzaparin in injecting drug users is feasi- ble and appears to result in satisfactory clinical responses.

The possibility of concomitant infection in injecting drug users with venous thrombosis should always be addressed, as it appears to be a common phenomenon. Early drainage of abscesses and antimicrobial chemotherapy, often administered intramuscularly or orally because of lack of peripheral venous access, is central to the appropriate care of these patients.

(Postgrad Med f 2000;76:561-565)

Keywords: iliofemoral venous thrombosis; intravenous drug abuse; groin abscess; low molecular weight heparin

In recent years there has been a dramatic increase in recreational drug misuse in the North East of Scotland. ${ }^{1}$ The number of new patients reported to the Drug Misuse Database at Grampian Health Board has increased from 98 in 1991/92 to 1178 in 1997/98 (Information and Statistics Division, NHS in Scotland, personal communication). Heroin use represents a major ongoing public health problem in the area and is reflected in the fact that the number of new clients attending the Needle Exchange Centre in Aberdeen has increased from 34 in 1991 to 624 in 1998 (Drugs Action, Aberdeen, personal communication).

The risks of infection associated with injecting drug use are well known and include soft tissue infection, abscess formation, transient bacteraemia ("a dirty hit"), right and left sided endocarditis, hepatitis due to the B, C, D and $G$ viruses, and HIV infection. ${ }^{2}$ Arterial occlusion is another well described hazard, ${ }^{3}$ but there are only three reports in the English language literature of iliofemoral venous thrombosis occurring as a complication of drug injection into the groin. ${ }^{4-6}$ The vascular complications of injecting drug use tend to arise after prolonged drug misuse, with its attendant superficial thrombophlebitis and consequent lack of easy peripheral venous access. The committed injecting drug user ultimately attempts to administer drugs into a deep vein-usually in the groin, with the potential 
for both vascular injury and deep abscess formation. ${ }^{3}$

Between 1994 and 1999 we treated 20 patients with iliofemoral venous thrombosis. All were committed injecting drug users without peripheral venous access. The use of low molecular weight heparin (LMWH) was an attractive option in these circumstances due to its proved efficacy in treatment for deep vein thrombosis, ease of administration, and lack of any need for monitoring coagulation tests. Its successful use therefore seemed worthwhile reporting.

\section{Methods}

A computer search of the hospital records was undertaken for all patients with a deep venous thrombosis admitted to the Aberdeen Infection Unit over a 10 year period (June 1989 to June 1999). Most of the patients have been seen in the recent past. The case notes of those patients who were injecting drug users were further examined and data were collected on age, sex, duration of injecting habit, site of deep vein thrombosis, confirmatory tests, treatment given, the presence of abscesses and coexistent bacteraemia, pulmonary embolism, and infection with hepatitis $B$ virus ( $\mathrm{HBV})$, hepatitis $\mathrm{C}$ virus (HCV), and HIV.

\section{Results}

The principal clinical characteristics and investigational features of the 20 consecutive patients identified with injection related iliofemoral venous thromboses are summarised in table 1. One patient was admitted in 1994, two in 1997, 11 in 1998, and six up to June 1999. The median age was 28 years (range 22-43 years) and the male to female ratio 13:7. All patients had been regularly injecting heroin before admission. The first patient (1994) had also injected temazepam and two others (1997) amphetamine. The median duration of injecting for the group was 6.5 years (range 2-17 years). Two of the patients had previously been diagnosed with injection related iliofemoral venous thromboses in the other limb, one and two years respectively.

All of the deep venous thromboses were confirmed using Doppler ultrasound and occurred in the iliofemoral segment, eight on the right, 11 on the left, and one bilaterally. All patients were right handed. One woman was only ever injected by her partner.

Nine of the patients were bacteraemic on admission. The organisms isolated were: Staphylococcus aureus (4); $S$ aureus and group B streptococcus (2); $S$ aureus and group G streptococcus (1); S aureus and Streptococcus intermedius (1); and Streptococcus pyogenes (1). Other associated conditions included groin abscess on white cell and ultrasound scanning (9), soft tissue infection of the groin, thigh, and lower abdomen (4) and upper limb (2), $S$ aureus osteomyelitis (1), and pulmonary embolism (1). Pus from four abscesses grew $S$ aureus; one also contained mixed anaerobes while five were sterile.

Anti-HCV antibody was positive in all of the 18 patients tested (two patients refused to be tested). Anti-HIV antibody was negative in the 13 patients tested (seven refused). Tests for current or previous infection with $\mathrm{HBV}$ (HBsAg or anti-HBcoreAb) were positive in five patients and negative in 13; two patients refused to be tested.

Only five of the 20 patients were receiving methadone as part of an opiate substitution/ harm reduction programme before their admission to the Infection Unit. Of the 15 patients not receiving methadone, 13 accepted methadone while in hospital and continued it after discharge in collaboration with the general practitioner (GP) and the Regional Drug Problems Service.

\section{ANTICOAGULANT MANAGEMENT}

Eighteen patients were treated with subcutaneous LMWH (tinzaparin 175 units/kg once daily); three of them received intravenous unfractionated heparin initially for one, 10 and 12 days respectively. The first patient (1994)

Table 1 Patient characteristics and details of therapy

\begin{tabular}{|c|c|c|c|c|c|c|c|c|}
\hline $\begin{array}{l}\text { Year of } \\
\text { presentation }\end{array}$ & Age (years) & Sex & $\begin{array}{l}\text { Duration of } \\
\text { injection (years) }\end{array}$ & Associated conditions & $\begin{array}{l}\text { Hospital therapy } \\
\text { (days) }\end{array}$ & $\begin{array}{l}\text { Home therapy } \\
\text { (weeks) }\end{array}$ & $\begin{array}{l}\text { Total therapy } \\
\text { (weeks) }\end{array}$ & $\begin{array}{l}\text { Outcome } \\
\text { (3 months) }\end{array}$ \\
\hline 1994 & 25 & $\mathrm{~m}$ & 8 & Nil & Heparin (3) & Nil & 0 & Cure \\
\hline 1997 & 31 & $\mathrm{~m}$ & 16 & Nil & Heparin (7) & Tinzaparin (4) & 5 & Swollen \\
\hline 1997 & 30 & $\mathrm{f}$ & 5 & STI/bacteraemia & Tinzaparin $^{*}(12)$ & Tinzaparin (10) & 12 & Swollen \\
\hline 1998 & 25 & $\mathrm{~m}$ & 2 & Osteomyelitis & Tinzaparin (26) & Tinzaparin (8) & 12 & Cure \\
\hline 1998 & 31 & $\mathrm{~m}$ & 11 & Abs/bacteraemia & Tinzaparin (8) & Tinzaparin (6) & 7 & Swollen \\
\hline 1998 & 32 & $\mathrm{~m}$ & 17 & Bacteraemia & Tinzaparin (14) & Tinzaparin (4) & 6 & Swollen \\
\hline 1998 & 23 & $\mathrm{~m}$ & 3 & $\mathrm{PE} / \mathrm{Abs} /$ bacteraemia & Tinzaparin $^{\dagger}(40)$ & Warfarin (26) & 26 & Lost \\
\hline 1998 & 43 & $\mathrm{~m}$ & 17 & Nil & Tinzaparin (14) & Tinzaparin (2) & 4 & Cure \\
\hline 1998 & 24 & $\mathrm{f}$ & 2 & Nil & Tinzaparin (10) & Tinzaparin (2) & 4 & Lost \\
\hline 1998 & 22 & $\mathrm{f}$ & 5 & Nil & Tinzaparin (3) & Tinzaparin (12) & 12 & Swollen \\
\hline 1998 & 33 & $\mathrm{f}$ & 10 & Abs & Tinzaparin ${ }^{\ddagger}(14)$ & Tinzaparin (6) & 8 & Swollen \\
\hline 1998 & 36 & $\mathrm{~m}$ & 4 & STI groin & Tinzaparin (15) & Nil & 2 & Cure \\
\hline 1998 & 26 & $\mathrm{~m}$ & 3 & Nil & Nil & Nil & 0 & Swollen \\
\hline 1998 & 23 & $\mathrm{f}$ & 4 & Abs/bacteraemia & Tinzaparin (4) & Tinzaparin (12) & 12 & Swollen \\
\hline 1999 & 34 & $\mathrm{~m}$ & 14 & $\mathrm{Abs} / \mathrm{b}$ acteraemia & Tinzaparin (21) & Tinzaparin (6) & 9 & Cure \\
\hline 1999 & 35 & $\mathrm{f}$ & 10 & Bacteraemia & Tinzaparin (7) & Tinzaparin (2) & 3 & Lost \\
\hline 1999 & 24 & $\mathrm{~m}$ & 6 & Abs/bacteraemia & Tinzaparin (14) & Tinzaparin (6) & 8 & Cure \\
\hline 1999 & 25 & $\mathrm{f}$ & 7 & Abs & Tinzaparin (7) & Tinzaparin (5) & 6 & Cure \\
\hline 1999 & 30 & $\mathrm{~m}$ & 7 & Abs & Tinzaparin (11) & Nil & 2 & Cure \\
\hline 1999 & 26 & $\mathrm{~m}$ & 3 & Abs/bacteraemia & Tinzaparin (21) & Tinzaparin (9) & 12 & Lost \\
\hline
\end{tabular}

Abs: groin abscess; PE: pulmonary embolism; STI: soft tissue infection

* Received intravenous heparin for 10 days then LMWH for two days; $†$ received intravenous heparin for 12 days then LMWH for 28 days; $\ddagger$ received intravenous heparin for one day then LMWH for 13 days. 
was treated with unfractionated heparin by intravenous infusion, but took his own discharge after three days. Another patient took his discharge against medial advice before starting treatment.

The initial hospital treatment with LMWH or unfractionated heparin $(n=19)$ was for a median duration of 10.5 days (range 3-40). In three patients the leg swelling had resolved and treatment been discontinued before discharge (unfractionated heparin three days, LMWH 11 days and LMWH 15 days, respectively). LMWH was continued after discharge in 15 patients, the duration of treatment being determined pragmatically, depending on the severity of the thrombosis and the patient's anticipated compliance with therapy. The median duration of post-discharge LMWH therapy in these patients was 6.5 weeks (range $2-12$ weeks). The median total duration of LMWH treatment was seven weeks $(n=17)$. Thirteen patients self administered the drug as prescribed, and two attended either the ward or their GP practice for daily injections. The patient who had a pulmonary embolism was subsequently treated with warfarin for three months.

In the six months after discharge, nine of 14 patients were readmitted to hospital with drug injection related problems (six patients had been out of hospital for $<6$ months). In the 12 months after discharge 10 of 12 patients were readmitted with injection related problems (eight patients have been out of hospital for $<12$ months). One patient developed a second iliofemoral venous thrombosis in the same leg and two of the group presented with a second thrombosis in the other leg within 12 months of the first episode, suggesting continued injecting drug misuse in the groin in spite of our exhortations.

At recent review ( $>3$ months after discharge) eight patients were asymptomatic (that is, without leg pain or swelling) but seven had a chronically swollen post-phlebitic leg. Repeat Doppler ultrasound scan was performed in two patients with the greatest leg swelling. There was no clot in one patient and a persisting nonocclusive clot in the other. None had suffered a pulmonary embolism, as judged clinically by the presence of respiratory symptoms or signs; formal imaging was not performed. Five patients have been lost to follow up. From a review of the local register of deaths none of these patients had died during the study or since its completion.

\section{INFECTION MANAGEMENT}

Nine of our patients had a groin abscess, two had groin or thigh and lower abdominal soft tissue infections, and nine of the 20 were bacteraemic. All but one of the groin abscess required surgical drainage. One patient underwent radical debridement of necrotic tissue in the thigh with skin grafting and a prolonged stay in the intensive therapy unit.

Where venous access was achievable, patients were treated with intravenous antimicrobials active against staphylococci and strepto- cocci, for example, benzylpenicillin and flucloxacillin. Where venous access was impossible, because of thrombosed superficial veins, patients were treated with intramuscular teicoplanin. If suspected clinically, Gram negative bacilli were treated with oral ciprofloxacin and anaerobes with oral metronidazole. All abscesses were successfully treated in this way and none of the patients with bacteraemia developed endocarditis. The patient with osteomyelitis of the tibia required protracted antimicrobial therapy.

\section{Discussion}

Deep venous thrombosis after intravenous drug use has been described previously. Seven patients in the USA developed upper limb deep venous thrombosis after injecting cocaine, ${ }^{4}$ four patients in the UK reported lower limb deep venous thrombosis after injecting temazepam (part of a larger series on the complications of temazepam abuse), ${ }^{5}$ and 14 patients in Australia reported lower limb deep venous thrombosis after injecting methadone (part of a larger series on the complications of methadone injection). ${ }^{6}$ There have been three other small case series in the non-English literature: Brazil, six patients, ${ }^{7}$ Spain, three patients, ${ }^{8}$ and Norway, four patients. ${ }^{9}$ This series represents the largest to date and is the first to describe the use of LMWH in treatment.

In our study the age of our patients (median 28 years) and the duration of their injecting "career" (median 6.5 years) suggest that iliofemoral venous thrombosis occurs after many years of self injection, when available superficial veins have become thrombosed. All the deep vein thrombosis in this group of patients occurred in the iliofemoral segment, presumably because of ease of injection at this site.

All of those in our cohort who were tested for HCV proved to be seropositive, reflecting the high incidence of this infection among drug users locally. There were 764 new HCV positive tests at the virus laboratory in Aberdeen between 1991 and 1998, 60\% of which were in injecting drug users (Virus Laboratory, Aberdeen Royal Infirmary, personal communication). None of those tested for HIV was positive, reflecting the low prevalence of this infection in North East Scotland. ${ }^{10}$

LMWHs, such as tinzaparin, have largely replaced intravenous unfractionated heparin in the treatment of peripheral deep venous thrombosis. LMWH has been shown to be as effective as conventional unfractionated heparin, has no general requirement for therapeutic monitoring, and has a convenient once or twice daily subcutaneous dosage regimen which the patient may self administer ${ }^{11}$ making it convenient for long term outpatient usage.

LMWH must be used with caution in those with severe renal impairment as the drug is excreted by the kidney. ${ }^{12}$ In such circumstances it is advisable to monitor antifactor Xa levels and adjust the dose accordingly. LMWH may 
cause heparin induced thrombocytopenia and, although it is less likely to do so than unfractionated heparin, ${ }^{13}$ it is advisable to monitor the platelet count for the first 10 days of treatment. The risk of osteoporosis is possibly less with LMWH than with unfractionated heparin. ${ }^{14}$ Treatment with warfarin after initial anticoagulation with $\mathrm{LMWH}$ is inappropriate for injecting drug users due to their chaotic lifestyle, problems in controlling and monitoring the international normalised ratio, noncompliance with prescribed treatment, and poor venous access.

Fourteen of our 17 patients self administered the tinzaparin after discharge for between two and 12 weeks - but no information is available regarding full compliance with the treatment prescribed. It is difficult to assess the success of treatment with LMWH in our patients, as persistent leg swelling may represent either unresolved thrombosis or repeated ongoing injecting behaviour. Of the 15 patients who were reviewed after discharge, eight were asymptomatic and seven had a chronically swollen leg. None suffered clinical pulmonary embolism, the most important consequence of deep vein thrombosis. It raises the question of whether more prolonged treatment is necessary, perhaps 8-12 weeks.

These retrospective data suggest that an appropriate approach to management of deep vein thrombosis in this group is the use of self injected LMWH after initial hospital treatment. Patients should be encouraged to continue treatment for at least six weeks, and possibly up to 12 weeks in severe cases, although this may not be achieved. It is unlikely that a controlled clinical trial could be accomplished in this patient cohort.

The issue of lifelong anticoagulation with LMWH for injecting drug users with recurrent deep vein thrombosis who continue to inject has been raised. Clearly the optimal approach is to help the user to stop injecting - through involvement in an opiate substitution programme if necessary. Should the injecting drug user continue to inject we would not recommend lifelong anticoagulation with LMWH as we feel the risks may outweigh the benefits of such a strategy.

If therapeutic anticoagulation is contraindicated (for example due to a high bleeding risk), inferior vena cava (IVC) filter insertion (for example with a Greenfield caval filter) should be considered..$^{15} \mathrm{~A}$ recent trial suggests that an initial protective effect from an IVC filter may be balanced by a later increased risk of recurrent deep vein thrombosis. ${ }^{16}$ The utilisation of temporary filters may reduce this risk, and are under evaluation. ${ }^{17}$ The insertion of an IVC filter was not considered in any of our patients as none had a contraindication to anticoagulation. To our knowledge, there are no reports in the literature of IVC filters being employed in injecting drug users with deep vein thromboses.

Infection in the involved groin was the cause of significant morbidity in our cohort of patients. The groin was also the commonest site affected in a series of 145 hospitalisations

\section{Learning points}

- Vascular complications of injection drug misuse, notably venous thromboses, tend to arise after some years of injecting (median 6.5 years)

- Treatment with tinzaparin appears to result in satisfactory outcomes and should be continued for six weeks or more

- Iliofemoral venous thrombosis in injecting drug users is commonly accompanied by groin abscess, soft tissue infection, and bacteraemia

- Early drainage of abscesses with appropriate antimicrobial therapy may prevent complications, including endocarditis

of 89 Swiss injecting drug users with acute soft tissue infections and abscesses. ${ }^{18}$ In that study serious complications occurred in 17 patients, and four lower extremity amputations were undertaken because of arterial occlusion. None of our patients required amputation.

$S$ aureus and various streptococci were the main pathogens identified in our patients-a finding similar to other reports. ${ }^{19} 20$ Aggressive treatment of these infections with surgical drainage and appropriate antimicrobials therapy resulted in a satisfactory outcome in all patients.

The possibility of concomitant infection in injecting drug users with venous thrombosis should always be considered as it appears to be a common phenomenon. It is now our policy to employ ultrasound, white cell scanning, and echocardiography in their screening.

We are grateful to Luan Bruce at Drugs Action, Aberdeen and Elaine Parry at Trinity Park House, Edinburgh for providing the local data on drug use. We also thank Dr Pamela Molyneaux, Consultant Virologist, for kindly providing the local data on hepatitis $\mathrm{C}$ virus infections.

1 Dykhuizen RS, Brunt PW, Atkinson P, et al. Ecstasy induced hepatitis mimicking viral hepatitis. Gut 1995;36:939-41.

2 Brettle RP. Infection and injection drug use. F Infect 1992;25:121-31.

3 Woodburn KR, Murie JA. Vascular complications of injecting drug misuse. Br f Surg 1996;83:1329-34.

4 Lisse JR, Davis CP, Thurmond-Anderle ME. Upper extremity deep venous thrombosis: increased prevalence due to cocaine abuse. Am $\mathcal{F}$ Med 1989;87:457-8.

5 Ruben SM, Morrison C. Temazepam misuse in a group of injecting drug users. Br $\dot{\mathcal{F}}$ Addict 1992;87:1387-92.

6 Darke S, Ross J, Hall W. Prevalence and correlates of the injection of methadone syrup in Sydney, Australia. Drug Alcohol Depend 1996;43:191-8.

7 Bertoncini RL, Silveira PG, Ceola L, et al. [Vascular compliBertoncini RL, Silveira PG, Ceola L, et al. [Vascular compli-
cations in intravenous drug addicts] [Portuguese]. Rev Assoc Med Bras 1992;38:204-8.

8 Valencia ME, Adrados M, Moreno V, et al. [Deep venous thrombosis of the upper limb in IVDA patients with HIV infection: a report of 3 cases (letter) [Spanish]. An Med Interna 1994;11:467-8.

9 Jensenius M, Holm B, Calisch TE, et al. [Deep venous thrombosis in intravenous drug addicts] [Norwegian]. Tidsskr Nor Laegeforen 1996;116:2556-8.

10 Mackenzie AR, Laing RBS, Urbaniak SJ, et al. Epidemiology and outcome of HIV infection in North-East Scotland (1985-1997). F Infect 1999;38:107-10.

11 Bick LR, Haas SK. International consensus recommendations. Summary statement and additional suggested guidelines. Med Clin North Am 1998;82:613-33.
lionsted guide12 Weitz JI. Low-molecular weight-heparins. N Engl f Med 1997;337:688-98.

13 Warkentin TE, Levine MN, Hirsh J, et al. Heparin-induced thrombocytopenia in patients treated with low-molecularweight heparin or unfractionated heparin. $N$ Engl f Med 1995;332:1330-5. 
14 Monreal M, Lafoz E, Olive A, et al. Comparison of subcutaneous unfractionated heparin with a low molecular weight heparin (Fragmin) in pro boembolism and contraindications to coumarin. Thromb Haemost 1994;71:7-11.

15 Hyers TM, Hull RD, Weg JG. Antiothrombotic therapy for venous thromboembolism. Chest 1995;108:335-51s.

16 Decousus H, Leizerovicz A, Parent F, et al. A clinical trial of vena cava filters in the prevention of pulmonary embolism in patients with proximal deep-vein thrombosis. $N$ Engl f Med 1998;338:409-15

17 Tai NRM, Atwal AS, Hamilton G. Modern management of pulmonary embolism. Br f Surg 1999;86:853-68.
18 Henriksen BM, Albrektsen SB, Simpler LB, et al. Soft tissue infection from drug abuse. A clinical and microbiological review of 145 cases. Acta Orthop Scand 1994;65:625-8.

19 Schnall SB, Holtom PD, Lilley JC. Abscesses secondary to parenteral abuse of drugs. A study of demographic and bacteriological characteristics. F Bone foint Surg Am 1994;76: 1526-30.

20 Summanen PH, Talan DA, Strong C, et al. Bacteriology of skin and soft-tissue infections: comparison of infections in intravenous drug users and individuals with no history of intravenous drug use. Clin Infect Dis 1995;20 (suppl 2):S279-82. 